\title{
Relación dialógica entre el profesorado senior y el profesorado novel universitario
}

\author{
Maria Luisa Rodríguez Moreno
}

Universidad de Barcelona - Facultad de Pedagogía (Spain)

doi: 10.7358/ecps-2015-012-rodr

luisarodriguez@ub.edu

\section{DIALOGIC RELATIONSHIP BETWEEN JUNIOR AND SENIOR UNIVERSITY PROFESSORS \\ RELAZIONE DIALOGICA TRA PROFESSORI UNIVERSITARI JUNIORS E SENIORS}

\begin{abstract}
The intergenerational relation in the European universities has evolved over the years. In the beginning was characterized for a hierarchical structure until more democratic situation. In this article is studied the dialogical relation between junior and senior professors to facilitate the learning of teaching and the adjustment to new critical moments. The author proposes the analysis of expectations, needs and projects for better orientation to the young professors and give practical suggestions about effective mentoring in Spanish universities. To ensure the teacher ability of socialization or adaptation to the real context of the university structure, which involves an inevitable dialectic based on expectations over the medium to long term, in line with the paradigm of socialization interactive, through which seniors and juniors teachers accepte mutually influences and changes. This will lead to a gradual and imperceptible enrichment, by which different cohorts of students will benefit. At the same time, advances in the development of mentoring novice teacher will be an endless source of ideas for applied research.
\end{abstract}

Keywords: Expectations, Dialogical relationship, Junior professor, Mentoring, Senior professor. 


\section{INTRODUCCIÓN}

Leemos en Reggio (2010) que el aprendizaje es el producto de una acción relacional y dialógica. El aprendizaje dialógico resulta de un diálogo igualitario en el que diferentes personas (profesor y alumno, alumno/alumno, mentor/protegido, por ejemplo) intercambian argumentos basados en el razonamiento y no en las relaciones de poder. Aprender desde el diálogo es la mejor manera de transformar la sociedad. La actividad transformadora de la persona que aprende se produce a través de la relación que consigue establecer con el mundo y con los otros. Aprender es un modo de salir del mundo cerrado de la persona, suficiente para sí mismo pero inconcluso; a través de la experiencia del tú, el yo de la persona que aprende reconoce y construye significados y toma forma. "Cuando se dice Tú, se dice al mismo tiempo el $Y_{o}$ del par verbal Yo-Tú» (Buber, 1969). Buber coincide con Freire enque la falta de diálogo impide la comprensión, la construcción de mundos y en que es funcional sólo cuando reproduce el conocimiento (Freire,1970).

Partiendo de nuestra postura ante la relación dialógica como la más idónea, iniciamos este trabajo, diciendo que desde finales de los ochenta algunos autores europeos vienen reflexionando sobre la iniciación a la docencia (teacher induction), pero se han centrado más en la enseñanza primaria y secundaria que en los niveles universitarios. La inducción - introducción o iniciación - viene definida como el "proceso de transición de quien estudia para ser profesor hasta el logro de la autogestión profesional» y es igualmente importante para el profesorado sin experiencia que con ella (Letvin, 1992). La iniciación del profesorado en su actividad docente es considerada por Burke (1987) mucho más que el breve período introductorio a la primera experiencia docente. Iniciar al profesorado novel o junior es un largo y comprometido proceso que exige planificar estudios y programas educativos propios de las instituciones universitarias, organizar prácticums en aulas de nivel superior para observar su dinámica, facilitar experiencias fuera y dentro del ámbito educativo y entrenarse en la toma de decisiones que progresivamente les vayan acercando correctamente a las exigencias reales de su puesto de trabajo.

Iniciarse es, en realidad, parte de un proceso continuado que termina con el máximo desarrollo de un profesional. La mayoría de los autores coinciden en subdividir en grandes fases la transición de persona no experimentada a experimentada. Fases tales como, por ejemplo, transitar de alumno a profesor; de ser persona idealista a ser realista; de desconocer las normas institucionales a aprender los roles de un funcionario; deno conocer a los estudiantes a descubrirsu verdadera identidad, etc. Y fases más complejas de socialización múltiple que van desde la exploración de las funciones profesionales hasta su 
consolidación, con toda una serie de problemas concurrentes (vid. para más detalle la tesis doctoral de Bozu, 2008).

El itinerario vital y laboral de alguien que desea ser profesor (docente e investigador) es parecido al de cualquier profesional y se extiendedesde el inicio en la profesión hasta la jubilación; no obstante, en términos prácticos, algunos autores convienen en aceptar que los siete primeros ańos de ejercicio en el magisterio son los que más van a influir en el desarrollo profesional de un docente (Vonk \& Schars, 1987). De estos siete ańos, el más investigado es el primero, pues se considera un período de prueba. Los procesos más estudiados, por ser los que emergen en esos primeros ańos de ejercicio, se refieren al crecimiento personal del profesorado novel, a su profesionalización o adquisición de experiencia y a sus relaciones con el medio donde debe actuar.

Con referencia a estos tres procesos, creo que hay algunas fuerzas institucionales que pueden influir significativamente desde sus propias estructuras. Por una parte, la atención que profesores senior, grupos de investigación e institutos de ciencias de la educación puedan prestar a las necesidades del profesorado novel. Por otra, los mecanismos de supervisión y evaluación de la calidad docente, ya sea desde los mismos departamentos universitarios, ya sea desde las agencias de evaluación de la calidad a niveles superiores, estatales o independientes. En este caso conviene aclarar que sería bueno diagnosticar la competencia del profesor novel no sólo para transmitir conocimientos, sino para acercarse al grupo de sus estudiantes transfiriendo con eficacia su conocimiento. Asimismo evaluar su competencia paracomunicarse, planificar las materias, trabajar en equipo con la plantilla profesoral y otras habilidades igualmente valorables. Finalmente, velar por la capacidad de socialización o adaptación al contexto real de la estructura universitaria, que implica una inevitable dialéctica: o asumir las normas institucionales (con la consiguiente construcción de las expectativas a medio y largo plazo) o acogerse al paradigma de una socialización interactiva, a través de la cual seniors y juniors se influyen y aceptan mutuamente cambios y proyectos.

\section{Cómo EJERCER LA PROFESIÓN EN LA UNIVERSIDAD}

La relación intergeneracional en la universidad española se ha caracterizado hasta finales del siglo XX por una estructura muy jerárquica en que el/la catedrático/a creaba su escuela, compuesta por la cátedra (silla, cadeira, cadira) y un pintoresco conjunto de profesores/as de menor categoría (ayudantes, adjuntos, agregados, titulares, etc.), que iban siguiendo las directrices docentes e investigadoras del que se consideraba jefe o líder natural. A partir del final 
de los años noventa, las cosas han ido cambiando progresivamente hacia una democratización de las relaciones departamentales aunque, a la vez, todo hay que decirlo, hacia una dispersión algo desorientadora en los planes docentes y las líneas de investigación. Desde los informes de 2002, referentes al Espacio Europeo de Educación Superior (Informe 24/05/2002) y del proyecto Tunning, de 2003, producto de las recomendaciones de la Comunidad Europea («El futuro de la educación hasta 2010»), se han ido construyendo las bases de una nueva educación universitaria caracterizada, muy especialmente, por promover nuevos objetivos y nuevas funciones (Rodríguez-Moreno, 2006). Objetivos que priorizan el aprendizaje de nuevas competencias, nuevas habilidades y nuevas responsabilidades, iniciando de este modo un camino hacia replanteamientos más cercanos al humanismo, a la promoción de nuevos saberes y al meta-aprendizaje (aprender a aprender).

A este estado de cosas hay que añadir que, a partir de las recomendaciones de Bolonia y del sunami que ha supuesto la prejubilación y jubilación de mucho profesorado ordinario (en España), estamos asistiendo a la emergencia de una nada desestimable colectividad de profesorado novel poseedor de nueva savia y de nuevas técnicas más cercanas a la sociedad del conocimiento. Este profesorado novel ya no padece la anterior estructura jerárquica, obsoleta, de organización de las plantillas universitarias, sino que colabora en los Departamentos y Facultades en igualdad de condiciones, de objetivos y de trato. El rol del colectivo de catedráticos o profesorado titular ya no es tan directivo, y las funciones del profesorado jubilado - senior y/o emérito - son, sobre todo, de apoyo. Se abren nuevas perspectivas, más claras y más eficaces en la formación y seguimiento del desarrollo de la carrera del profesor junior. Y, por tanto, en la investigación de sus necesidades, expectativas y proyectos.

\section{UN FUTURO MUY CERCANO}

He citado tres de los aspectos que creo más urgentes para los proyectos futuros de investigación y de formación: diagnosticar las expectativas, atender a las necesidades que ésas han originado y favorecer y estimular los proyectos del profesorado (tanto novelcomo senior). Estos colectivosviven sus correspondientes crisis de transición (el choque ante la realidad - el reality shock, de Veenman, 1984) en términos personales y académicos, de modo que ese shock podría ofrecer diferentes temas a investigar: cómo el profesorado novel (en traumática transición) percibe sus problemas; cómo cambia sus conductas, actitudes y personalidad; cómo se replantea su puesto de trabajo; y a qué causas atribuye el enfrentamiento a la realidad. Veenman, en 1984 aportaba 
en un exhaustivo estudio varias investigaciones al respecto (aunque no del profesorado universitario) que pueden servir de punto de referencia para investigaciones en la docencia universitaria.

El mismo Veenman, doce años más tarde (1996) afirmaba que, a pesar de que todos los gobiernos hablan de lo interesante que es alcanzar la calidad en los sistemas educativos, la iniciación del profesorado novel sigue siendo una asignatura pendiente. De tal manera que, al no existir programas de formación para los profesores de nuevo cuño, muchos de ellos abandonan la docencia en los tres primeros años. La investigación sobre las actuaciones y problemas del profesorado novel ha sido escasa y ha estado centrada, como antes hemos expuesto, en la primaria y la secundaria; aunque a partir de las innovaciones promovidas por la Comisión Europea y los drásticos cambios sugeridos en el plan Bolonia, en esta última década se inician trabajos (a veces tímidos) al respecto, más descriptivos que prescriptivos. Véanse, por ejemplo, los trabajos de Goodlad, 1997; y los de los españoles Cruz, 1999; Marcelo et al., 1993 - que elaboraron un cuestionario sobre la iniciación a la docencia universitaria; Colén et al., 2000, y Feixas, 2002.

Estoy convencida de que el profesorado novel precisa de programas de iniciación y de que seniors y noveles se pueden ayudar y enriquecer mutuamente. De hecho, sea cual sea la clasificación, en fases o etapas, el proceso evolutivo de unos y otros (con sus semejanzas y sus diferencias), nos sugiere el siguiente gráfico genérico, con connotaciones de continuum:

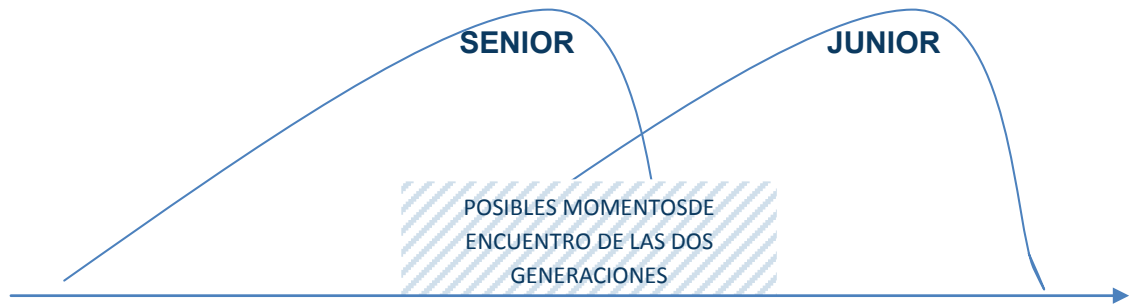

En el gráfico queda claro que siempre pueden darse generosos y didácticos encuentros entre seniors y juniors, que serán tanto más eficaces si los Departamentos y las Facultades asumen la necesidad de sinergia y se prestan a planificar acciones formativas e investigadoras, con la correspondiente propuesta de recursos y metodologías (sin olvidar que cada profesor/a senior o junior son casos singulares y genuinos). Es imposible, por tanto, generalizar, ya que cada universidad tiene sus propias y definitorias características, las transiciones son distintas entre personas y edades, y los estilos cognitivos y personales de transferencia del saber y de la experiencia son irrepetibles. 


\subsection{El profesorado senior}

En España siempre ha habido mucho interés por mejorar la docencia en las aulas universitarias. Desde los años noventa, el profesorado universitario tiene a su disposición agencias evaluadoras de la calidad de su trabajo, tanto a nivel nacional como a nivel autonómico e incluso internacional. Los productos de su labor investigadora, sus publicaciones y sus innovaciones muestran también que la carrera del profesor universitario es competitiva siempre en busca de la calidad máxima. El profesor seniores el que ha pasado por todas las vicisitudes de un arco iris de la vida profesional que abarca desde los primeros momentos de duda y de esfuerzo (a veces, sólo basados en el ensayo y el error que, en ciertos momentos, han parecido baldíos) hasta su fase de establecimiento como funcionario ordinario en la estructura universitaria y su época dorada de impartición de sus propias ideas y de sus propios hallazgos (fruto, frecuentemente de la investigación aplicada). Desde finales del siglo XIX hasta el inicio del siglo XXI, el profesor universitario ha vivido una carrera algo monolítica y rectilínea, llevado por ciertas rutinas (con sus pros y sus contras, por supuesto), y bastante centrado en el paradigma de profesor funcionario. La expresión más genuina y popular decía que la cátedra era vitalicia.

\subsection{El profesorado novel o junior}

Como ya he comentado, hoy día los modelos y las estructuras han ido cambiando y el profesorado novel ya no se desenvuelve en una dinámica tan rígida y ordenada. A lo largo de los años el concepto de profesor novel ha ido cambiando y evolucionando al igual que la enseñanza universitaria. Actualmente, según Feixas (2000), vemos que el profesorado novel, en la etapa universitaria, es profesorado joven, con alguna experiencia profesional y con menos de 3 ó 5 años de experiencia docente en una institución universitaria. Bozu (2009) apunta que los profesores universitarios noveles inician su carrera docente y sus primeras experiencias en la universidad como posgraduados, dentro de un programa de doctorado; es el caso de los becarios predoctorales, de los ayudantes en las universidades españolas y de los graduate teaching assitants en Europa y EEUU. Durante este periodo formativo, los estudiantes de doctorado tienen que trabajar como docentes principiantes, para ofrecer soporte en la docencia a los profesores titulares y experimentados del departamento. Esta etapa está acompañada de la angustia que padecen todos los noveles por el hecho de saber si serán capaces de enfrentarse a la realidad y, al mismo tiempo, si lo harán mejor que aquellos profesores que tuvieron y que 
no fueron de su agrado. Al mismo tiempo, este sufrimiento se caracteriza por las carencias que padecen los noveles:

- El acceso a una plaza permanente y la promoción académica.

- La competitividad en el ámbito de las publicaciones, asistencia a congresos, seminarios y jornadas.

- La necesidad de incorporar la teoría y la práctica a sus enfoques de la enseńanza para motivar alalumnado y fomentar su interés por la materia y por el aprender mediante metodologías didácticas y vivenciales.

- La importancia del establecimiento de redes y de ser reconocido como miembro de la comunidad teniendo en cuenta el contexto institucional.

Estas necesidades se pueden satisfacer a partir de los tímidos programas de formación inicial del profesorado novel propuestos en algunas universidades españolas. Según Fernández (2008), los modelos más innovadores, siguiendo el paradigma del profesional reflexivo, se centran en programas basados en la reflexión sobre la propia práctica y la revisión colectiva de las actuaciones docentes. Uno de los programas pioneros y con más trayectoria es el programa implementado y desarrollado por el Instituto de Ciencias de la Educación de la Universidad Politécnica de Valencia con el objetivo de iniciar y desarrollar el proceso de profesionalización del docente universitario en el siglo XXI mediante las competencias pedagógicas básicas que promueve el programa.

A partir de estas dos situaciones, se imponeun proceso de reflexión compartida entre seniors y juniors (Vélaz de Medrano, 2009). Pero no es tarea fácil, por los problemas que plantea; del desencuentro entre estas dos categorías o clases de profesorado, los más expertos y los menos expertos, hemos deducido, considerando simultáneamente nuestras experiencias y las opiniones de los teóricose investigaciones, las siguientes carencias y peculiaridades:

a. Se ha dado más atención a la formación del profesorado novel en los niveles de primaria y secundaria. El profesorado novel universitario no ha recibido, en general, atención específica institucionalizada.

b. No se detectan programas universitarios concretos, sólidos y afianzados de iniciación del profesorado junior a la docencia y a la investigación. La orientación personal y académica en algunas instituciones ha substituido lo que podrían ser planes estructurados; y mucho menos se dan investigaciones acerca de metodologías de iniciación.

c. Distintos investigadores (tanto desde la metodología cuantitativa como cualitativa) han propuesto o señalado que el paso del rol de graduado a profesor novel se produce en fases, longitudinales o evolutivas, entre las que la más estresante es la primera, que suele durar de 6 a 7 ańos. Este estrés o shock ante la realidad acaba, muchas veces, en el abandono.

d. La transición desde la calidad de graduado a profesor novel es estresante, difícil y de cierta complejidad psico social, por los esfuerzos que exige a 
nivel personal, familiar y académico. El profesorado novel sobrevive, la mayoría de las veces, por ensayo y error.

e. También hemos conocido (y está demostrado) que la transición de profesor con experiencia a senior jubilado es, igualmente, estresante.

f. Se impone racionalizar y conectar ambas transiciones para mejorar los procesos transitorios de ambos colectivos.

Por estas razones, iniciada la segunda década del segundo milenio, conviene $-\mathrm{y}$ urge - indagar acerca de varias dimensiones de la función docente del profesorado novel, para edificar sobre nuevos fundamentos y paradigmas la formación futura de los idóneos profesores universitarios. Podría ser pertinente distribuir esas funciones para su mejor análisis (Tabla 1).

En el mundo académico se constata que el profesorado novel universitario suele iniciarse simplemente a partir de sus experiencias vitales, de sus conocimientos formales y no formales y de sus cambios en la conceptualización de qué es su profesión y cómo ha de actuar en ella. Nadie se ha ocupado o se está ocupando de formarle para la docencia.

Tabla 1. - Ámbitos de posible pesquisa en la transición y desarrollo del profesorado novel (basado en Rodríguez, López, Pol, \& Pascual, 2012).

\section{ÁMBITOS A INVESTIGAR EN LA TRANSICIÓN \\ Y DESARROLLO DEL PROFESORADO NOVEL UNIVERSITARIO}

\section{EXPECTATIVAS}

\section{Necesidades}

\section{Proyectos}

1. Aprender contenidos

2. Aprender a transferir conocimientos y motivar al alumnado

3. Conocer metodologías didácticas generales y diferenciales. Planificar y organizar los contenidos

4. Conocer estrategias de orientación personal, tutoría y orientación profesional

5. Conocer las normas institucionales y la política educativa del contexto

6. Innovar

7. Evaluar y mantener disciplina y colaboración en las aulas

8. Investigar y publicar

9. Trabajar en equipo
- Cambiar actitudes y conductas

- Econsiderar el autoconcept y emociones

- Planificar, seleccionar recursos según objetivos

y competencias, en equipo

- Replantearse la competitividad individual con otros colegas

- Aprender a innovar y a rebelarse (del conservadurismo a lo progresista)

- Profundizar en la calidad de la expresión de las ideas científicas (en publicaciones)
- Participar activamente en proyectos de Investigación y desarrollo de ámbito nacional

- Seguir realizando producciones científicas en forma de artículos y comunicaciones

- Investigar en temas actuales y de interés para favorecer el desarrollo profesional dentro de la comunidad universitaria

- Pertenecer y promocionarse en el equipo de investigación que colaboro

- Pertenecer a un grupo de investigación que permita participar en proyectos relacionados con el ámbito de la orientación académica y profesional 
El uso de la mentoría sería (entre otras, como el portafolios o carpeta docente, el seek, la supervisión individualizada de la adquisición de competencias, los métodos heurísticos, los prácticums probatorios o experienciales, etc.) una metodología interesante y útil en los tiempos que corren, sobre todo porque hay, actualmente, mucho profesorado senior vocacionaly con suficiente potencial para colaborar en la iniciación del junior. De hecho, la mentoría se ha considerado muy importante en el acompañamiento del alumnado universitario (Valverde et al., 2003-2004), pero no se ha prestado excesiva atención al profesorado novel o en proceso de iniciación.

\subsection{Etapas por las que transita el profesor novel mientras va pasando a senior}

El profesor novel (beginning teacher, novice, novel) inicia su andadura desde un punto concreto (al acabar sus estudios de postgrado, la mayoría de las veces) hasta pasados 5 o 7 ańos de experiencia, dolorosa y complicada. El proceso de aprender a ser profesor es de gran complejidad e implica conexión con otros profesores más experimentados y contextos multivariados, condicionados por las culturas de cada universidad. La docencia, en esa primera etapa, exige que el profesorado sea competente en su tarea inicial y futura y consiga las metas finales en condiciones adversas o difíciles; por ejemplo, cuando el alumnado presenta diferentes capacidades y niveles, cuando las condiciones del contexto no sean predecibles con facilidad, cuando los controles políticos y administrativos sean rígidos e inamovibles, cuando los sistemas educativos y curriculares varíen con demasiada frecuencia o cuando las expectativas de docentes y discentes sean inestables. Burke (1987, p. viii) considera que esos condicionamientos son más un reto que una barrera. De alguna manera, el profesorado novel se va a ver abocado a transitar por diferentes etapas como las que propone Vonk (1994, p. 88), que nos parecen prácticas y oportunas (Tabla 2):

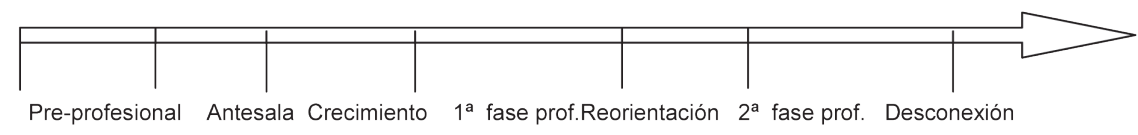


Tabla 2. - Descripción de las etapas del desarrollo profesional del profesor novel.

ETAPAS

BREVE DESCRIPCIÓN DE LAS ETAPAS

Pre-profesional

Antesala

Crecimiento

$1^{\text {a }}$ fase profesional

Reorientación

$2^{\text {a }}$ fase profesional

Desconexión
Período de formación inicial, centrado en lo personal y lo ambientaly en la cristalización y ampliación de los conocimientos propios de las materias que ha de enseñar..

Fase de transición a través de un umbral, es decir, primer año de experiencia docente, por el que el profesor novel intenta sobrevivir bajo nuevas condiciones y empieza a vislumbrar su identidad profesional y su propio repertorio de actividades

Período entre el segundo y el séptimo año de servicios. Son aspectos clave: aprender a ominar el aula (motivar, evaluar y controlar el avance del alumnado) y reflexionar sobre los errores o aciertos de la propia manera de enseñar y educar.

Varios ańos transcurridos - unos 5, a partir del sexto o séptimo año de experiencia - hasta la madurez docente, en que coincide la experiencia adquirida de varias dimensiones (enseñar contenidos, evaluar resultados, crear recursos, dialogar con profesionales afines) con el descubrimiento de las técnicas de investigación y aplicación de la teoría y el intercambio científico con profesores e investigadores muchas veces a nivel internacional.

Crisis a mitad de la carrera profesional y vital. Momentos en que todo lo aprendido y aplicado se pone en cuestión y se reinicia toda una campaña auto aplicada de análisis de los propios errores y de los propios aciertos. Estas crisis, muchas veces coinciden con fases de evaluación cualitativa y cuantitativa por parte de los gobiernos y/o de las administraciones universitarias sobre el personal docente de más alto nivel: Es lo que se ha venido a denominar control de la calidad y evaluación de los méritos.

Período vital y profesional en que se puede calificar al profesor de verdaderamente experto, no sólo por el nivel de sus conocimientos teóricos sino también por sus habilidades investigadoras, docentes y divulgadoras. Fase que coincide con el estatus adquirido en la sociedad del conocimiento y en la recogida de todo lo sembrado en los 25/30 años anteriores. El profesor, ya senior, puede iniciar sus primeras actividades de mentoría con profesorado novel.

Período previo a la jubilación, con multitud de variantes, en función del bagaje de la persona, de sus intereses universitarios y extrauniversitarios y de su colaboración con colegas nacionales e internacionales. 


\section{LA MENTORÍA}

A lo largo de estas últimas dos décadas se ha publicado interesante bibliografía acerca de la mentoría y se han desarrollado jornadas al respecto (Jackson, 1990; Clutterbuck \& Megginson, 1995; Furlong \& Maynard, 1995; Mullen, \& Lick, 1999; Blanc, 2003; Soler, 2003; Blanc \& Cuerrier, 2007; Molina, 2008; Velasco et al., 2009; Taveira, 2011). En general la mentoría es considerada un método para transferir los conocimientos prácticos de una persona a otra. Vonk (1994, 1996), Healey y Welchert (1990) la definen como «una relación dinámica y recíproca - en un ambiente laboral - entre una persona experimentada (mentor) y un aprendiz o novel (protegido) dirigida a promocionar el desarrollo profesional de ambos». Los profesores que se inician están interesados en recibir ayuda de una persona experta para adquirir identidad profesional y desarrollarsedesde el inicio hasta el total desarrollo profesional; y, por su parte, el interés del mentor es que se refleje en el protegido el propio repertorio de acciones. Casi siempre, en los profesores más mayores, la relación de mentoría significa practicar de una manera generativa, emulando, de alguna manera el rol de la responsabilidad formativa de un padre. Siempre y cuando en esa fórmula se dé una auténtica reciprocidad. La relación de mentoría, de hecho enriquece el desarrollo profesional de las dos personas implicadas en ella.

Para funcionar como un correcto mentor, éste ha de poseer ciertas competencias (conocer lo que el principiante sabe y cuáles son los problemas que le preocupan, manejar las relaciones interpersonales y poseer ciertas competencias técnicas cercanas a la orientación personal - aconsejar, observar, responder, instruir, evaluar, etc.). De hecho, el mentor debe estar alerta en una serie de aspectos clave de sus protegidos: observar el proceso de su desarrollo general, profundizar en la naturaleza de los problemas (siempre dependientes de la época y del contexto) y coadyuvar al despliegue de teoría y práctica en el ejercicio de su incipiente profesión.La competencia relacional de un mentor a la hora de acompañar al protegée o profesor novel puede ser examinada desde los cuatro clásicos enfoques: el de acompańamiento no directivo, el colaborativo, el de asesoramiento y el de control. El primero es el más aconsejable por lo que comporta de escucha de las emociones del profesor novel, de la discusión sobre sus puntos fuertes y débiles y de evaluación del contacto o proceso comunicativo. Es innegable, y en ello coinciden muchos de los teóricos de la mentoría antes citados, que el profesor senior en sus roles de mentor, debe dominar ciertas técnicas como las de ser un excelente observador, la de devolver la síntesis de los datos recogidos, la de instructor o, finalmente, la de evaluador; siempre desde una perspectiva positiva y optimista (Galton \& Moon, 1994).

El rol de la persona tutorizada (o sujeto de la mentoría) radica, fundamentalmente, en su capacidad de escucha y sus expectativas. De hecho, desde 
las indicaciones de las competencias clave sugeridas por la Comisión Europea, el profesor novel debe demostrar (o ir adquiriendo) el máximo grado de competencia para la comunicación, para el aprendizaje continuado, parar relacionarse, trabajar en equipo, socializarse, planificar, negociar y transferir el conocimiento, así como para poner en práctica nuevas iniciativas con creatividad y confianza en sí mismo. La puesta en práctica de un exhaustivo balance de competencias personal y profesional será condición muy importante antes de solicitar la ayuda voluntaria de su mentor (Rodríguez, Serreri, \& Del Cimmuto, 2010).

\section{SugerenCias PARA UNA EFICAZ MENTORÍA}

Del análisis y discusión posterior de los itinerarios presentados por una profesora senior y tres profesores noveles (dos profesoras y un profesor) al Seminario de Sevilla 2012 (Rodríguez et al., 2012) sugerimos algunas acciones de mentoría, categorizadas en tres áreas: expectativas, necesidades y proyectos.

Si la mentoría está planificada con rigory atiende a las expectativas y necesidades de ambos protagonistas se produce una simbiosis y cambio progresivo de roles. Se dará el mutuo enriquecimiento («muta me, meta mu, tu eres yo, yo soy tu»), progresivo e imperceptible, del que se beneficiarán no sólo la institución sino también las diferentes cohortes de alumnado. A la vez, el avance en la preparación y mentoría del profesor novel será una fuente inagotable de ideas para la investigación aplicada. Parece necesario ofrecer sugerencias para la posible relación protegée/mentor cara a racionalizar el proceso de mentoría.

\subsection{Sugerencias para orientar las expectativas del profesorado novel}

\begin{tabular}{|c|c|}
\hline $\begin{array}{c}\text { EXPECTATIVAS } \\
\text { DEL PROFESORADO NOVEL }\end{array}$ & $\begin{array}{c}\text { ALGUNAS ACCIONES SUGERIDAS } \\
\text { DEL/DE LA MENTOR/A }\end{array}$ \\
\hline Aprender contenidos & $\begin{array}{l}\text { Facilitar documentación, sobre todo } \\
\text { de los clásicos en la especialidad y enseñarle } \\
\text { a buscarla con raciocinio y rigor }\end{array}$ \\
\hline $\begin{array}{l}\text { - Aprender a transferir conocimientos } \\
\text { y motivar al alumnado } \\
\text { - Conocer metodologías didácticas } \\
\text { generales y diferenciales } \\
\text { - Planificar y organizar los contenidos }\end{array}$ & $\begin{array}{l}\text { Proveerle de metodologías y competencia } \\
\text { didáctica; métodos activos, socializados, } \\
\text { individualizados, de proyectos, y de enseñanza } \\
\text { aprendizaje a distancia }\end{array}$ \\
\hline
\end{tabular}


Conocer estrategias de orientación personal, tutoría y orientación profesional

- Conocer las normas institucionales y la política educativa del contexto

- Evaluar y mantener disciplina y colaboración en las aulas

Investigar e innovar

Publicar
Iniciarle en los fundamentos y filosofía de la orientación personal y profesional, para que aprenda a ser un buen tutor de sus alumnos en clases de diferente tamaño

Colaborar con la institución para poner en marcha su normativa, estatutos, métodos de control y evaluación cualitativ y cuantitativa, siempre desde un espíritu crítico

Dirigir o codirigir la tesis doctoral del profesor novel; seńalar los paradigmas de la creatividad y de la aplicación de los resultado de la investigación a la realidad profesional; apoyar y colaborar en la investigación básica; enseñar a crear recursos genuinos

Iniciarle en todo lo relativo a las exigencias formales y de contenido de las publicaciones científicas en sus múltiples formativos y plataformas, para que redacte exitosamente

\subsection{Sugerencias para orientar las necesidades del profesorado novel}

\section{Necesidades \\ DEL PROFESORADO NOVEL \\ Algunas ACCIONES SUGERIDAS \\ DEL/DE LA MENTOR/A}

Dedicar mucho tiempo al estudio (conseguir conciliaciones varias)

Cambiar actitudes (de lo ideal a lo real, reconsiderando utopías e ilusiones)

Cambiar conductas (de la exigencia a la adaptación, de la rigidez a la flexibilidad)
Exponer la mejor manera de preparase; coadyuvar a la búsqueda de contenidos y de bibliografía; acercar a las instituciones las reivindicaciones para la conciliació de la profesora novel con tu vida familiar

Enseñar cómo enfocar de la carrera profesional de una manera realista, analizando los pros y los contras de actitudes excesivamente soñadoras; caso frecuente al entrar en la universidad

Exponer metodologías didácticas más flexibles y adaptadas a la tipología del alumnado; el profesor novel, al estar más inseguro, tiende a la rigidez docente en las aulas 
Reconsiderar el auto concepto y emociones (control de los estreses y desequilibrios)

Planificar, seleccionar recursos según objetivos y competencias, en equipo

Replantearse la competitividad individual con otros colegas

Aprender a innovar y a rebelarse (del conservadurismo a lo progresista)

Profundizar en la calidad de la expresión de las ideas científicas (en publicaciones)
Explicar cómo enfrentarse a los bloqueos; coadyuvar con el profesor en momentos de cansancio, crisis o miedo

Ayudar a organizar los planes de las asignaturas, a ubicarlas en el conjunto del currículum de las titulaciones y a desgranar las competencias idóneas en los programas

Mediar en los casos de enfrentamiento demasiado explícito y enseñar a relativizar las situaciones de una mal entendida competitividad; exponer las ventajas del trabajo en equipo

En los grupos de investigación, saber crear espacios de encuentro con las ideas, de reflexión teórica y de reacción a la rutina y la desidia; explicar cómo de una actividad cotidiana pueden surgir multitud de ideas innovadoras

Colaborar con los profesores noveles en la redacción, presentación y edición de artículos, capítulo de libros, artículos, recensiones, blogs, páginas web de difusión de la investigación, explicando las mejores maneras de redactar y comunicarse con nivel y dignidad

\subsection{Sugerencias para orientar los proyectos del profesorado novel}

\begin{tabular}{|c|c|}
\hline $\begin{array}{c}\text { PROYECTOS } \\
\text { DEL PROFESORADO NOVEL }\end{array}$ & $\begin{array}{l}\text { ALGUNAS ACCIONES SUGERIDAS } \\
\text { DEL/DE LA MENTOR/A }\end{array}$ \\
\hline $\begin{array}{l}\text { Participar activamente en proyectos } \\
\text { de investigación y desarrollo de ámbito } \\
\text { nacional }\end{array}$ & $\begin{array}{l}\text { Solicitar la colaboración del junior en grupos } \\
\text { de trabajo }\end{array}$ \\
\hline $\begin{array}{l}\text { Seguir realizando producciones científicas } \\
\text { en forma de artículos y comunicaciones }\end{array}$ & $\begin{array}{l}\text { Incluir en las publicaciones } \\
\text { de los investigadores senior aspectos } \\
\text { redactados por el profesorado junior }\end{array}$ \\
\hline $\begin{array}{l}\text { Acudir a Congresos, Jornadas, Seminarios, } \\
\text { etc., relacionados con la orientación } \\
\text { académica y profesional para intercambiar } \\
\text { experiencias }\end{array}$ & $\begin{array}{l}\text { Facilitar su matriculación a eventos nacionales } \\
\text { e internacionales con inclusión del junior } \\
\text { en comunicaciones y posters }\end{array}$ \\
\hline
\end{tabular}


Investigar en temas actuales y de interés para favorecer el desarrollo profesional dentro de la comunidad universitaria

Pertenecer y promocionarse en el equipo de investigación en que se colabora
Incluir a los investigadores principiantes en proyectos comunitarios, sociales, educativos y políticos

Contar con la ayuda activa e inclusión oficial del junior en los equipos de investigación (nacionales y de ámbito europeo)

Conseguir becas predoctorales para así iniciar Auxiliar en la solicitud administrativa estudios de doctorado y burocrática de ayudas y becas

En definitiva, una interesante y urgente labor está por hacer. La solicitud de ideas aportadas por los docentes universitarios va a ser muy apreciada, en estos momentos de cambio y de estructuras mucho más globalizadas. Conviene creer en el futuro.

\section{ANEXos}

Síntesis de las respuestas recibidas en un cuestionario dirigido a profesorado universitario de orientación. III Seminario de la Red Interuniversitaria de Profesorado de Orientación. RIPO y MIDE de la Universidad de Sevilla, Sevilla, 22-23 de Marzo 2012 (nota: en muchos casos no se indicó la Universidad de procedencia).

\section{Anexo I}

Respuestas a la pregunta: ¿Qué expectativas crees que tiene el profesorado novel en tu centro?

En este momento muy difíciles por la crisis. Pero a corto plazo creo que muy buenas, por la necesidad de incorporar gran cantidad de personal docente e investigador a las facultades de Educacion. (U. Lleida)

Del 1 al $10=3$

Formarse como investigadores y docentes.

Estabilizar (afianzar) su puesto de trabajo.

Desarrollarse profesionalmente y personalmente. (U. Huelva)

Aprender de todo y de todos.

Realización de la tesis.

Publicar en revistas de impacto.

Pertenecer a grupos de investigación. (U. Huelva) 
Cumplir criterios exteriores en un mundo competitivo.

Ser docente-investigador competente.

Participar en equipos de investigación cooperativos.

Acceder a una plaza de docente universitario. (UCM)

Solo puedo hablar desde lo que yo puedo creer, pues siempre es complejo saber lo que piensa cada persona. No se si hay un colectivo «novel» como tal. Dicho esto, creo que ven el panorama muy «negro». Los consejos que le llegan desde otros con más experiencias es que deben publicar, publicar y publicar en revistas de impacto. Es decir, que todo lo que hagan les sirva para el CV académico por encima de todo. $\mathrm{Y}$ a la vista están las intervenciones de hoy. (U. Sevilla)

Hay que distinguir entre dos grupos: el profesor novel propiamente dicho, que se inicia en esta carrera desde una beca y que acaba de titularse; y el profesor asociado que es un colectivo emergente por la política de nuestras universidades. Estos no son «junior» y sus expectativas en la universidad, en la mayoría de los casos, se limitan a «echar unas horas» para sacar un sobresueldo. Por lo tanto, centrándose en el primero de los grupos, creo que sus expectativas son, sobre todo, integrarse en la vida universitaria (investigación y docencia) y aprender todo lo necesario para desenvolverse en ella. (U. Sevilla)

En primer lugar "consolidar» un puesto de trabajo Formarse como docentes e investigadores. (U. Valladolid)

Creo que tienen ilusión, esperanzas y muchas ganas de aprender. Aspectos del quehacer diario de un profesor universitario que a los que llevamos tiempo a veces se nos olvida. Esta savia nueva nos renueva y hace que no perdamos de vista donde estamos. (U. Barcelona)

\section{Anexo II}

Respuestas a la pregunta: ¿Qué necesidades crees que tiene el profesorado novel en tu centro?

Formación docente. Creo que los aspectos de investigación ya están cubiertos con la incorporación a equipos de investigación, para su carrera docente. Pero no reciben ni formación ni acompańamiento en su labor de profesores/as en el aula. (U. Lleida)

Seguridad laboral.

Consciencia de la esencia de ser profesor.

Adiestramiento para navegar en el mar de las acreditaciones, investigaciones, evaluaciones de calidad, ...

Investigar para generar conocimientos además de publicar.

Sobrevivir a las TIC. (U. Huelva)

Salidas al extranjero.

Cursos de formación.

Conocimiento del mundo profesional.

Formación permanente. (U. Huelva) 
Afectivas- seguridad.

Conocimiento situacional.

Sentido de pertinencia y equipo.

Aprender a decir no. (UCM)

Considero que están tan "perdidos» que las necesidades van en todos los ámbitos: personales, didácticos, metodológicos, de investigación, capacidad para hablar en publico, ... Acordes a que aprenden "poco» en la carrera y que deben trabajar mucho por «recuperar» lo que antes no hicieron por diferentes motivos. (U. Sevilla)

Las inmediatas: relativas a ir generando un CV que les permita la transición en sus carreras profesionales.

Aprender: sobretodo, en investigación; para ser autónomos en el futuro, necesitan integrarse activamente en proyectos que les permitan desarrollar habilidades $y$, a veces, la confianza necesaria para ir haciendo sus propias aportaciones.

Guía: la universidad es un mundo claro, complejo, ... no hay que aprender por ensayo-error si otras personas pueden ofrecer apoyo y acompańamiento. (U. Sevilla)

Formación para la docencia universitaria.

Participación en proyectos de investigación.

Estrategias de investigación, publicación, ... (U. Valladolid)

Apoyo, formación y ánimos. Esto solo se conseguirá trabajando colaborativamente e incorporándolos en nuestros trabajos. Facilitando y guiándolos en su proyecto profesional. (U. Barcelona)

\section{Anexo III}

Respuestas a la pregunta: ¿Qué funciones podría realizar el profesorado senior a favor del profesorado novel?

En ambos campos:

Docente: realizando mentorías en su intervención en el aula.

Investigación: coaching en su proyecto profesional y personal. (U. Lleida)

Acompañar señalando caminos posibles, inculcando motivación y sosiego, impulso y serenidad, tutelar dejando hacer (incluso equivocarse), pellizcar en prisas. Dialogar, conversar, ilusionar, ...

La experiencia no se puede aprender, hay que vivirla. Necesita tiempo y trabajo. (U. Huelva)

Mentor, guía, orientador, tutor, asesorar sobre las posibles publicaciones. (U. Huelva)

Mediador y facilitador (información, material docente, fuentes bibliográficas, procesos

de investigación, ....).

Integrador.

Contacto con otros investigadores.

Feedback proactivo. (UCM) 
Abrir oportunidades sin cerrar puertas (esto tiene que ver con problemas de poder, egoísmo, etc.).

Dedicar tiempo a escuchar las dudas, incertidumbres, problemas, ... del novel.

Ofrecer oportunidades para trabajar en proyectos y publicar.

Ayudar al novel a aprender. (U. Sevilla)

Proporcionar oportunidades de aprendizaje: a una persona joven puede resultarle difícil que le concedan un proyecto; para ir haciendo un CV. Y aprendiendo a investigar necesita que le ofrezcan oportunidades para aprender.

Animarle a tener iniciativas, orientarle para que saque mayor tipo a su trabajo, ... nunca dirigirle, sino dejar que se «equivoque» y que se desarrolle como profesional a partir de sus experiencias.

«Presentarle» en la sociedad académica. (U. Sevilla)

Orientación para la estructura y difusión de la investigación. (U. Valladolid)

En estos momentos los que somos ya seniors no debemos repetir los errores que han cometido y cometen con nosotros. Las actitudes dinámicas de investigación - contar con todos/as y de los equipos docentes facilita este intercambio y formación. (U. Barcelona)

\section{REFERENCIAS}

Blanc, M., \& Cuerrier, Ch. (2007). Le mentorat en politique auprès des femmes. Un mode d'acompagnement prometteur. Montréal: Les Éditions du Remue-Ménage.

Cuerrier, Ch. (2003). Pour alimenter la réflexion. Le mentorat et le développement professionnel. Québec: Fondation de l'Entrepreneuship, Col. Mentorat, Doc. 13.

Bozu, Z. (2008). La carpeta docente como práctica formativa y de desarrollo profesional del profesorado universitario novel. Un estudio de casos. Tesis de Doctorado inédita. Barcelona: Universitat de Barcelona. Vid. también: El profesorado universitario novel: estudio teórico de su proceso de inducción o socialización profesional. Revista Electrónica de Investigación y Docencia (REID), 3, Enero, 2010, 55-72. http://www.revistareid.net/revista/n3/REID3art3.pdf.

Bozu, Z. (2009). El profesorado universitario novel y su proceso de inducción profesional. Revista Internacional de Investigación en Educación, 2, 317-328.

Buber, M. (1969). Yo y tu. Trad. de Horacio Crespo. Madrid: Nueva Visión.

Burke, K. (1987). Teachers development. Induction, renewal and redirection. London: Falmer Press.

Clutterbuck, D., \& Megginson, D. (1995). Mentoring in action: A practical guide for managers. London: Kogan Page.

Colén, M. et al. (2000). Las necesidades formativas del profesorado novel para el ejercicio de la función docente. Comunicación al I Congreso Internacional Docència Universitària i Innovació. Barcelona: Universitat de Barcelona - ICE.

Cruz, M. A. (1999). El programa de formación inicial para la docencia universitaria de la Universidad Autónoma de Madrid. http://www.ub.edu/forum/Conferencias/sadu.htm. 
Feixas, M. (2002). El desenvolupament professional del professor universitari com a docent. Tesis de Doctorado inédita. Barcelona: Universitat Autónoma de Barcelona.

Fernández, A. (2008). La formación inicial del profesorado universitario: el título de Especialista Universitario en Pedagogía Universitaria de la Universidad Politécnica de Valencia. Revista Interuniversitaria de Formación del Profesorado, 63 $(22,3), 161-187$.

Freire, P. (1970). La pedagogía de los oprimidos. Madrid: Siglo XXI.

Furlong, J., \& Maynard, T. (1995). Mentoring student teachers. The grow of professional knowledge. London: Routledge.

Galton, M., \& Moon, B. (Eds.). (1994). Handbook of teacher training in Europe. London: David Fulton Publishers.

Granade, C. (1992). How to Mentor in the Midst of Change. Virginia: Association for Supervision and Curriculum Development.

Healey, C. C., \& Welchert, A. J. (1990). Mentoring relations: A definition to advance research and practice. Educational Researcher, 19(9), 17-22.

Letvin, E. (1992). Induction. In R. Fessler \& J. C. Christensen, The teacher career cycle (pp. 59-87). Boston: Allyn and Bacon.

Marcelo, G. (Coord.). (2001). La funcion docente. Madrid: Síntesis.

Molina, J. P. (2008). El «mentoring», práctica necesaria en las consultoras de comunicación y relaciones públicas. Anàlisi, 37, 151-160.

Mullen, C. A., \& Lick, D. W. (1999). New directions in mentoring. Creating culture of synergy. London: Falmer Press.

Reggio, P. (2010). El cuarto saber. Guía para el aprendizaje experiencial. Valencia: Ediciones del CREC. http://www.dva.gva.es/crec.

Rodríguez, M. L. (2006). Evaluación, balance y formación de competencias laborales transversales. Barcelona: Laertes.

Rodríguez, M. L., López, D., Pol, C., \& Pascual, L. (2012). La riqueza de la relación intergeneracional de investigadores/as de orientación. Proyectos, expectativas, necesidades. Ponencia presentada al III Seminario de la Red Interuniversitaria de Profesorado de Orientación. RIPO y MIDE de la Universidad de Sevilla, Sevilla, 22-23 Marzo 2012.

Rodríguez, M. L., Serreri, P., \& Del Cimmuto, A. (2010). Desarrollo de competencias. Teoría y práctica. Balance, proyecto profesional y aprendizaje basado en el trabajo. Barcelona: Laertes.

Soler, M. R. (2003). Mentoring. Estrategia de desarrollo de recursos humanos. Barcelona: Gestión 2000 - Epise.

Taveira, M. C. (2011). Mentoría y gestión personal de la carrera en contextos universitarios. I Jornadas Internacionales de transición y orientación a lo largo de la vida, Marzo 2011. Madrid: UNED.

Valverde, A., Ruiz, C., García, E., \& Romero, S. (2003-2004). Innovación en la orientación universitaria: la mentoría como respuesta. Contextos Educativos, 6-7, 87-112. 
Veenman, S. (1984). Preceived problems of beginning teachers. Review of Educational Research, 54(2), 143-178.

Velasco, P. J. et al. (2009). La mentoría entre iguales y el desarrollo de competencias. JIMCUE '09, IV Jornadas Internacionales Mentoring \& Coaching, Universidad Empresa, Madrid, 23-25 Noviembre 2009.

Vélaz de Medrano, C. (2009). Competencias del profesor-mentor para el acompañamiento al profesorado principiante. Revista de Curriculum y Formación del Profesorado, 13(1).

Vonk, J. H. (1994). Teacher induction: The great omission in education. In M. Galton \& B. Moon, Handbook of teacher training in Europe. Issues and trends (pp. 85-108). London: David Fulton.

Vonk, J. H. (1996). Conceptualizing the mentoring of beginning teachers. Paper presented at the Annual Conference of the American Educational Research Association, New York, 8-12 April 1996.

Vonk, J. H., \& Schras, G. A. (1987). From beginning to experienced teachers: A study of the professional development of teachers during their first four years of service. European Journal of Teacher Education, 10(1), 95-110.

\section{RiassunTO}

Il rapporto intergenerazionale nelle università europee si è evoluto nel corso degli anni. In principio è stato caratterizzato da una struttura gerarchica fino ad articolazioni recenti più democratiche. In questo articolo si studia la relazione dialogica tra docenti juniors e seniors per facilitare l'apprendimento dell'insegnamento e l'adeguamento ai momenti critici. L'autore propone l'analisi delle aspettative, dei bisogni e dei progetti per migliorare l'orientamento dei giovani professori attraverso un'attività di mentoring efficace da svilupparsi nelle università spagnole. Per garantire la capacità dell'insegnante di socializzazione o di adattamento al contesto reale della struttura universitaria, si propone lo sviluppo di una dialettica basata su aspettative a medio-lungo termine, in linea con il paradigma interattivo della socializzazione, attraverso il quale gli professori anziani e juniors accettano reciproche influenze e cambiamenti. Ciò porterà ad un arricchimento graduale e impercettibile, da cui le diverse coorti di studenti trarranno beneficio. Allo stesso tempo, i progressi nello sviluppo del mentoring dell'insegnante novizio costituirà una fonte inesauribile di idee per la ricerca applicata.

Parole chiave: Aspettative, Professore junior, Profesore senior, Relazione dialogica, Tutoraggio.

How to cite this Paper: Rodríguez Moreno, M. L. (2015). Relación dialógica entre el profesorado senior y el profesorado novel universitario [Dialogic relationship between junior and senior university professors]. Journal of Educational, Cultural and Psychological Studies, 12, 283-302. doi: 10.7358/ecps-2015-012-rodr 